\title{
Some Inequalities for the Omori-Yau Maximum Principle
}

\author{
Kyusik Hong \\ Korea Institute for Advanced Study, Hoegiro 85, Seoul 130-722, Republic of Korea \\ Correspondence should be addressed to Kyusik Hong; kszoo@kias.re.kr
}

Received 22 January 2015; Revised 25 June 2015; Accepted 2 July 2015

Academic Editor: Leszek Gasinski

Copyright (c) 2015 Kyusik Hong. This is an open access article distributed under the Creative Commons Attribution License, which permits unrestricted use, distribution, and reproduction in any medium, provided the original work is properly cited.

\begin{abstract}
We generalize A. Borbély's condition for the conclusion of the Omori-Yau maximum principle for the Laplace operator on a complete Riemannian manifold to a second-order linear semielliptic operator $L$ with bounded coefficients and no zeroth order term. Also, we consider a new sufficient condition for the existence of a tamed exhaustion function. From these results, we may remark that the existence of a tamed exhaustion function is more general than the hypotheses in the version of the Omori-Yau maximum principle that was given by A. Ratto, M. Rigoli, and A. G. Setti.
\end{abstract}

\section{Introduction}

Let $(M, g)$ be a smooth complete Riemannian manifold of dimension $n$. For a smooth real-valued function $h$ on $M$, a second-order linear differential operator $L: C^{\infty}(M) \rightarrow$ $C^{\infty}(M)$ without zeroth-order term can be written as

$$
L h=\operatorname{Tr}\left(A \circ \operatorname{Hess}_{h}\right)+g(V, \nabla h),
$$

where $A \in \Gamma(\operatorname{End}(\mathrm{TM}))$ is self-adjoint with respect to $g$, Hess $_{h} \in \Gamma($ End(TM)) is the Hessian of $h$ in the form defined by $\operatorname{Hess}_{h}(X)=\nabla_{X} \nabla h$ for $X \in \Gamma(\mathrm{TM})$, and finally $V \in \Gamma(\mathrm{TM})$. In this paper, we will deal with the semielliptic case, that is, $A$ is positive semidefinite at each point, and we always assume that

$$
\sup _{M} \operatorname{Tr}(A)+\sup _{M}|V|<\infty .
$$

Definition 1. A smooth complete Riemannian manifold $M$ is said to satisfy the Omori-Yau maximum principle for the Laplace operator $\Delta$ (the above semielliptic operator $L$ ) if for any $C^{2}$ function $h: M \rightarrow \mathbb{R}$ which is bounded from above and for any $\epsilon>0$ there is a point $x_{\epsilon} \in M$ such that $\left|h\left(x_{\epsilon}\right)-\sup _{M} h\right|<\epsilon,\left\|\nabla h\left(x_{\epsilon}\right)\right\|<\epsilon$, and $\Delta h\left(x_{\epsilon}\right)<\epsilon$ $\left(\operatorname{Lh}\left(x_{\epsilon}\right)<\epsilon\right)$.

The Omori-Yau maximum principle is a useful substitute of the usual maximum principle in noncompact settings. For the operator $\Delta$, Definition 1 is the well-known Omori-Yau maximum principle for the Laplacian, which was first proven by Omori [1] and Yau [2] when the Ricci curvature is bounded below. This was improved upon by Chen and Xin [3] and Ratto et al. [4] when the Ricci curvature decays were slower than a certain decreasing function tending to minus infinity. For instance, we have the following.

Theorem 2 (Ratto-Rigoli-Setti's condition [4, Theorem 2.3]). Let $o \in M$ be a fixed point and $r(x)$ be the distance function from $o$. Let one assumes that away from the cut locus of o one has

$$
\operatorname{Ricc}(\nabla r, \nabla r) \geq-(n-1) B G^{2}(r),
$$

where $B>0$ is some constant and $G(t)$ on $[0, \infty)$ satisfies

$$
\begin{aligned}
& \int_{0}^{\infty} \frac{1}{G(t)} d t=\infty, \quad G(0)=1, G^{\prime} \geq 0, \\
& \sqrt{G}^{(2 k+1)}(0)=0, \quad \forall k \geq 0, \\
& \limsup _{t \rightarrow \infty} \frac{t \sqrt{G(\sqrt{t})}}{\sqrt{G(t)}}<\infty .
\end{aligned}
$$

Then $M$ satisfies the Omori-Yau maximum principle for the Laplacian $\Delta$.

Borbély [5, Theorem] has given an elegant proof of the validity of the Omori-Yau maximum principle where 
the Ricci curvature condition (3) is replaced by the assumption $\Delta r(x) \leq G(r(x))$ without (4) and (5). Also, Bessa et al. [6, Theorem 5.6] proved Borbély's theorem [5, Theorem] for the $f$-Laplacian $\Delta_{f}$ for a selected smooth function on $M$. In this paper, we first show that Borbély's theorem [5, Theorem] is also true for our semielliptic operator $L$ by following his method in [5] (see Theorem 5).

To state other results, we need the following definitions.

Definition 3. Let $u$ be a real-valued continuous function on $M$ and let a point $p \in M$.

(i) A function $u$ is called proper, if the set $\{p: u(p) \leq r\}$ is compact for every real number $r$.

(ii) A function $v$ defined on a neighborhood $U_{p}$ of $p$ is called an upper-supporting function for $u$ at $p$, if the conditions $v(p)=u(p)$ and $v \geq u$ hold in $U_{p}$.

Definition 4. A proper continuous function $u: M \rightarrow \mathbb{R}$ is called a $\Delta$-tamed exhaustion, if the following condition holds:

(1) $u \geq 0$.

(2) At all points $p \in M$ it has a $C^{2}$ smooth, uppersupporting function $v$ at $p$ defined on an open neighborhood $U_{p}$ such that $\left\|\left.\nabla v\right|_{p}\right\| \leq 1$ and $\left.\Delta v\right|_{p} \leq 1$.

Royden [7] showed that every complete Riemannian manifold satisfying Omori-Yau's condition (i.e., the Ricci curvature is bounded from below) admits a $\Delta$-tamed exhaustion function. Inspired by Royden's article [7], Kim and Lee [8, Theorem 2] proved the Omori-Yau maximum principle for the Laplacian $\Delta$ when there exists a $\Delta$-tamed exhaustion function. Moreover, they proved that every complete Riemannian manifold satisfying Ratto-Rigoli-Setti's condition admits a $\Delta$ tamed exhaustion function [8]. Similar to Definition 4, we define an $L$-tamed exhaustion function (i.e., we replace $\Delta$ with $L$ ) [9, Definition 1.4]. Then, using the existence of an $L$-tamed exhaustion function, Hong and Sung [9, Theorem 2.1] generalized the Omori-Yau maximum principle for the Laplacian $\Delta$ to the operator $L$. In this paper, we give a new sufficient condition for the existence of an $L$-tamed exhaustion function (see Theorem 6). We prove this result using the ideas adapted from [8]. Note that Theorem 6, together with [9, Theorem 2.1], implies the maximum principle of Omori and Yau for the operator $L$. As a corollary, we prove that the existence of a $\Delta$-tamed exhaustion is more general than RattoRigoli-Setti's condition. Unfortunately, for the operator $L$, the relation between Borbély's condition (or the existence of an $L$ tamed exhaustion) and Ratto-Rigoli-Setti's condition remains for further study.

Now, we formulate our main results. From (1), $A$ is diagonalizable at each point on an orthonormal basis, since $A$ is symmetric. Then one can take a normal coordinate $\left(x_{1}, \ldots, x_{n}\right)$ around $x_{\epsilon} \in M$ such that $A$ at $x_{\epsilon}$ is represented as a diagonal matrix. Thus, we have

$$
\left.L h\right|_{x_{\epsilon}}=\left.\sum_{l} a_{l l}\left(x_{\epsilon}\right) \frac{\partial^{2}}{\partial x_{l}^{2}} h\right|_{x_{\epsilon}}+\left.\sum_{l} a_{l}\left(x_{\epsilon}\right) \frac{\partial}{\partial x_{l}} h\right|_{x_{\epsilon}},
$$

for a real-valued function $h$ on $M$, where each $a_{l l}\left(x_{\epsilon}\right)$ is nonnegative; the entries $a_{l l}\left(x_{\epsilon}\right)$ and $\left|a_{l}\left(x_{\epsilon}\right)\right|$ are bounded above as $x_{\epsilon}$ varies by (2). We introduce a locally defined differential operator for convenience as follows:

$$
\begin{aligned}
& \widetilde{\Delta}_{x_{\epsilon}}:=a_{11}\left(x_{\epsilon}\right) \frac{\partial^{2}}{\partial x_{1}^{2}}+\cdots+a_{n n}\left(x_{\epsilon}\right) \frac{\partial^{2}}{\partial x_{n}^{2}}, \\
& \widetilde{\nabla}_{x_{\epsilon}}^{1}:=a_{1}\left(x_{\epsilon}\right) \frac{\partial}{\partial x_{1}}+\cdots+a_{n}\left(x_{\epsilon}\right) \frac{\partial}{\partial x_{n}} \\
& \widetilde{\nabla}_{x_{\epsilon}}:=\left(a_{11}\left(x_{\epsilon}\right) \frac{\partial}{\partial x_{1}}, \ldots, a_{n n}\left(x_{\epsilon}\right) \frac{\partial}{\partial x_{n}}\right) .
\end{aligned}
$$

Put $d_{l}=a_{l l}\left(x_{\epsilon}\right)$ and $e_{l}=\left|a_{l}\left(x_{\epsilon}\right)\right|$ for $1 \leq l \leq n$. We may assume that $d_{1}$ and $e_{1}$ are the largest of $\left\{d_{1}, \ldots, d_{n}\right\}$ and $\left\{e_{1}, \ldots, e_{n}\right\}$, respectively.

Then we have the following.

Theorem 5. Let $o \in M$ be a fixed point and $r(x)$ be the distance function from $o$. Assume that for all $x \in M$

$$
\widetilde{\Delta}_{x} r(x) \leq G(r(x)),
$$

where $r$ is smooth, $r(x)>1$, and $G(t)$ on $[0, \infty)$ satisfies

$$
\int_{0}^{\infty} \frac{d t}{G(t)}=\infty, \quad G \geq 1, G^{\prime} \geq 0 .
$$

Then $M$ satisfies the Omori-Yau maximum principle for the operator $L$.

Theorem 6. Let $o \in M$ be a fixed point and $r(x)$ be the distance function from $o$. Assume that for all $x \in M$

$$
\widetilde{\Delta}_{x} r(x) \leq G(r(x)),
$$

where $r$ is smooth, $r(x)>1$, and $G(t)$ on $[0, \infty)$ satisfies

$$
\begin{aligned}
\int_{0}^{\infty} \frac{d t}{G(t)} & =\infty, \quad G \geq 1, G^{\prime} \geq 0, \\
\limsup _{t \rightarrow+\infty} & \frac{t \sqrt{G(\sqrt{t})}}{\sqrt{G(t)}}<+\infty .
\end{aligned}
$$

Then $M$ admits an L-tamed exhaustion function.

Remark 7. By [5, Corollary] and Theorem 6, Ratto-RigoliSetti's condition without $\sqrt{G}^{(2 k+1)}(0)=0 \forall k \geq 0$ implies the existence of a $\Delta$-tamed exhaustion function. Therefore, the existence of a $\Delta$-tamed exhaustion function for the conclusion of the Omori-Yau maximum principle for the Laplacian $\Delta$ is more general than the hypothesis in Theorem 2 .

There are some other sufficient conditions under which the Omori-Yau maximum principle for the Laplacian $\Delta$ holds [10-12]. Also, [13] deals with the general setting of semielliptic operators (trace type operators). Recently, Bessa and Pessoa [14, Theorem 1] present a sufficient condition for the conclusion of the Omori-Yau maximum principle 
for a second-order linear semielliptic operator with bounded first-order coefficients and no zeroth-order term. However, they will not consider the existence of a tamed exhaustion function as sufficient conditions for the conclusion of the Omori-Yau maximum principle.

\section{Proof of Theorem 5}

The proof is similar to the method in [5]. Let $U=\sup h$. We may assume that $h<U$ at every point of $M$; otherwise, $h$ has its maximum at some point and that point directly satisfies the Omori-Yau maximum principle for a semielliptic operator $L$.

Define the function $F(t)$ as

$$
F(t)=e^{\int_{0}^{t}(1 / G(s)) d s} .
$$

Then

$$
F^{\prime}=\frac{F}{G}
$$

Since $G \geq 1$ on $[0, \infty)$, we have $F \geq 1$, and $F^{\prime}>0$. Hence the function $F$ is strictly increasing, and $\lim _{t \rightarrow \infty} F(t)=\infty$. Since the set $\{x \in M: r(x) \leq 1\}$ is compact, we have

$$
U-\sup \{h(x): r(x) \leq 1\}>0 .
$$

For any positive constant $\epsilon<\min \{1, U-\sup \{h(x): r(x) \leq$ $1\}$, we define the function $h_{\lambda}: M \rightarrow \mathbb{R}$ as

$$
h_{\lambda}(x)=\lambda F(r(x))+U-\epsilon \text {. }
$$

Then

$$
h_{\lambda}(x)>h(x) \quad \text { if } r(x) \leq 1, \lambda \geq 0 .
$$

Because, for all $x \in M, F(r(x)) \geq 1$ and $U>h(x)$. If $\lambda>\epsilon$, then we have

$$
h_{\lambda}(x)>h(x), \quad \forall x \in M
$$

Define $\lambda_{0}$ as

$$
\lambda_{0}=\inf \left\{\lambda: h_{\lambda}(x)>h(x), \forall x \in M\right\} .
$$

Then, clearly, $\lambda_{0}>0$. Furthermore, we can obtain $h_{\lambda_{0}}(x) \geq$ $h(x)$ for all $x \in M$; that is, there is a point $x_{\epsilon} \in M$ such that $h_{\lambda_{0}}\left(x_{\epsilon}\right)=h\left(x_{\epsilon}\right)$. Assume that to the contrary $h_{\lambda_{0}}(x)>h(x)$ for all $x \in M$. Then we will show that there is a constant $\lambda^{\prime}$ with $\lambda_{0}>\lambda^{\prime}$ such that $h_{\lambda^{\prime}}(x)>h(x)$ for all $x \in M$. This is a contradiction to the definition of $\lambda_{0}$.

Let $\lambda_{0}>\lambda_{1}$. Because $\lim _{r \rightarrow \infty} F(r)=\infty$, there is a sufficiently large positive number $r_{0}$ such that $h_{\lambda_{1}}(x)>U>$ $h(x)$ for $r(x)>r_{0}$. Also, because the set $\left\{x \in M: r(x) \leq r_{0}\right\}$ is compact, the statement $h_{\lambda_{0}}(x)>h(x)$ for all $x \in M$ implies that there is a constant $\lambda_{2}$ with $\lambda_{0}>\lambda_{2}$ such that $h_{\lambda_{2}}(x)>h(x)$ for $r(x) \leq r_{0}$. Now, let $\lambda^{\prime}=\max \left\{\lambda_{1}, \lambda_{2}\right\}$. Then, for $\lambda_{0}>\lambda^{\prime}$, we have $h_{\lambda^{\prime}}(x)>h(x)$ for all $x \in M$. Moreover, by (17) and $\lambda_{0}>0$, we have $r\left(x_{\epsilon}\right)>1$.
Next, we have to show that $h_{\lambda_{0}}$ is smooth at $x_{\epsilon}$. Since $h_{\lambda}(x)=\lambda F(r(x))+U-\epsilon$, it is enough to show that $r$ is smooth at $x_{\epsilon}$. To avoid confusion, the point $o$, in the statement of Theorem 5, is switched to $p$. Note that $r$ is a Lipschitz function and is smooth on $M \backslash\left\{p, C_{p}\right\}$, where $C_{p}$ is the cut locus of $p$. Suppose that $x_{\epsilon} \in C_{p}$. Then we have two possibilities (Petersen [15, Lemma 8.2]); either there are two distinct minimizing geodesic segments $\gamma_{1}, \gamma_{2}:\left[0, t_{0}\right] \rightarrow M$ joining $p$ to $x_{\epsilon}$, or there is a geodesic segment $\gamma:\left[0, t_{0}\right] \rightarrow M$ from $p$ to $x_{\epsilon}$ along which $x_{\epsilon}$ is conjugate to $p$. Notice that

$$
t_{0}=r\left(\gamma_{i}\left(t_{0}\right)\right)=r\left(x_{\epsilon}\right) \text { for } i=1 \text { or } 2 \text {. }
$$

We consider the first case. Let $w=\gamma_{1}^{\prime}\left(t_{0}\right)$ and $v=\gamma_{2}^{\prime}\left(t_{0}\right)$. Since $\gamma_{1}$ and $\gamma_{2}$ are distinct segments, we have $w \neq v$. For $i=1$ or 2 , the functions $t \rightarrow r\left(\gamma_{i}(t)\right)$ are differentiable on $\left(0, t_{0}\right)$ and they have a left-derivative at $t_{0}$. Note that $h$ is $C^{2}$ smooth on $M$. From the definition of $\lambda_{0}, h_{\lambda_{0}} \geq h$, and $h_{\lambda_{0}}\left(x_{\epsilon}\right)=h\left(x_{\epsilon}\right)$ we obtain

$$
\liminf _{s \rightarrow 0^{+}} \frac{h_{\lambda_{0}}\left(\gamma_{2}\left(t_{0}+s\right)\right)-h_{\lambda_{0}}\left(\gamma_{2}\left(t_{0}\right)\right)}{s} \geq D_{v} h\left(x_{\epsilon}\right),
$$

where $D_{v} h\left(x_{\epsilon}\right)$ denotes the directional derivative of $h$ at the point $x_{\epsilon}$ in the direction of $v$. Furthermore, since $h_{\lambda_{0}}$ has a directional derivative at $x_{\epsilon}$ in the direction of $-v$, we have

$$
\begin{aligned}
-\lambda_{0} F^{\prime}\left(t_{0}\right) & =-\lambda_{0} F^{\prime}\left(r\left(x_{\epsilon}\right)\right)=D_{-v} h_{\lambda_{0}}\left(x_{\epsilon}\right) \\
& \geq D_{-v} h\left(x_{\epsilon}\right)=-D_{v} h\left(x_{\epsilon}\right) .
\end{aligned}
$$

This yields

$$
D_{v} h\left(x_{\epsilon}\right) \geq \lambda_{0} F^{\prime}\left(r\left(x_{\epsilon}\right)\right) .
$$

Hence, by (21) and (23), we get the following inequality:

$$
\begin{aligned}
& \liminf _{s \rightarrow 0^{+}} \frac{h_{\lambda_{0}}\left(\gamma_{2}\left(t_{0}+s\right)\right)-h_{\lambda_{0}}\left(\gamma_{2}\left(t_{0}\right)\right)}{s} \\
& \quad \geq \lambda_{0} F^{\prime}\left(r\left(x_{\epsilon}\right)\right) .
\end{aligned}
$$

Note that $\left(h_{\lambda_{0}}\left(\gamma_{2}\right)\right)^{\prime}=\lambda_{0} F^{\prime}\left(r\left(\gamma_{2}\right)\right) r^{\prime}\left(\gamma_{2}\right)$ and $r\left(\gamma_{2}\left(t_{0}\right)\right)=r\left(x_{\epsilon}\right)$. Recall that $\lambda_{0}>0$. Then, from (24), we can get

$$
\liminf _{s \rightarrow 0^{+}} \frac{r\left(\gamma_{2}\left(t_{0}+s\right)\right)-r\left(\gamma_{2}\left(t_{0}\right)\right)}{s} \geq 1
$$

The inequality (25) will lead to a contradiction. Since $\gamma_{1}$ and $\gamma_{2}$ are different segments, by connecting from the point $\gamma_{1}\left(t_{0}-\right.$ $s)$ to the point $\gamma_{2}\left(t_{0}+s\right)$ with a geodesic segment, there is a constant $c$ with $0<c<1$ such that, for a sufficiently small $s>0$, the distance $d\left(\gamma_{1}\left(t_{0}-s\right), \gamma_{2}\left(t_{0}+s\right)\right)<c 2 s$. Thus there is a constant $c^{\prime}$ with $0<c^{\prime}<1$ depending only on the angle of $v$ and $w$ such that

$$
r\left(\gamma_{2}\left(t_{0}+s\right)\right)<t_{0}+c^{\prime} s
$$

for a sufficiently small $s>0$. Note that $r\left(\gamma_{2}\left(t_{0}\right)\right)=t_{0}$. By plugging (26) to (25), we have a contradiction. 
From now, let us consider the second case. Since $\gamma$ is distance minimizing between $p$ and $x_{\epsilon}, r$ is smooth at $\gamma(t)$ for $0<t<t_{0}$. Let $m(t)=\Delta r(\gamma(t))$. Then $m(t)$ is also smooth for $0<t<t_{0}$. Because $\gamma\left(t_{0}\right)$ is conjugate to $p=\gamma(0)$ along $\gamma$, by a simple calculation, we get

$$
\lim _{t \rightarrow t_{0}^{-}} m(t)=-\infty
$$

Because $\lambda_{0} F^{\prime}\left(r\left(x_{\epsilon}\right)\right)>0$, by (23), we get $D_{v} h\left(x_{\epsilon}\right)>0$; that is, $\nabla h\left(x_{\epsilon}\right) \neq 0$. Hence the level surface $H=\{x \in M: h(x)=$ $\left.h\left(x_{\epsilon}\right)\right\}$ is a $C^{2}$ smooth hypersurface near $x_{\epsilon}$. Denote by $H_{s}$ the surface parallel to $H$ and passing through the point $\gamma\left(t_{0}-s\right)$ for some $s>0$. Since $H$ is $C^{2}$ smooth near $x_{\epsilon}$, the surface $H_{s}$ is also $C^{2}$ smooth near $\gamma\left(t_{0}-s\right)$ for a sufficiently small $s>0$. Therefore, by (27), for some sufficiently small $s$, the trace of the second fundamental form of $H_{s}$ at $\gamma\left(t_{0}-s\right)$ in the direction of $\gamma^{\prime}\left(t_{0}-s\right)$ is greater than $m\left(t_{0}-s\right)$, where $m\left(t_{0}-s\right)$ is the trace of the second fundamental form of the geodesic sphere $B\left(p, t_{0}-s\right)$ at $\gamma\left(t_{0}-s\right)$ with respect to the normal vector $\gamma^{\prime}\left(t_{0}-s\right)$. This implies that there has to be a point $q_{s} \in H_{s}$ sufficiently close to $\gamma\left(t_{0}-s\right)$, which lies inside $B\left(p, t_{0}-s\right)$; that is,

$$
r\left(q_{s}\right)<t_{0}-s
$$

Since $H_{s}$ is parallel to $H$, we also have a point on $q \in H$ such that the distance $d\left(q_{s}, q\right)=s$. By $(28)$, we have

$$
r(q)<t_{0}=r\left(x_{\epsilon}\right) \text {. }
$$

Since $F$ is strictly increasing, we get

$$
\begin{aligned}
h_{\lambda_{0}}(q) & =\lambda_{0} F(r(q))+U-\epsilon<\lambda_{0} F\left(r\left(x_{\epsilon}\right)\right)+U-\epsilon \\
& =h_{\lambda_{0}}\left(x_{\epsilon}\right)=h\left(x_{\epsilon}\right)=h(q) .
\end{aligned}
$$

This is a contradiction to the fact that $h_{\lambda_{0}}(x) \geq h(x)$ for all $x \in M$. Therefore, the function $r$ must be smooth at $x_{\epsilon}$.

By the definition of $F, F \geq 1, G \geq 1$, and $G^{\prime} \geq 0$, we have

$$
\begin{aligned}
0 & <F^{\prime}=\frac{F}{G}, \\
F^{\prime \prime} & =\frac{F^{\prime}}{G}-\frac{F G^{\prime}}{G^{2}}=\frac{F}{G^{2}}-\frac{F G^{\prime}}{G^{2}} \leq \frac{F}{G^{2}} .
\end{aligned}
$$

Because $\lambda_{0}>0, F \geq 1$, and $h\left(x_{\epsilon}\right)=\lambda_{0} F\left(r\left(x_{\epsilon}\right)\right)+U-\epsilon<U$, we have

$$
0<-\lambda_{0} F\left(r\left(x_{\epsilon}\right)\right)+\epsilon=U-h\left(x_{\epsilon}\right)<\epsilon .
$$

Hence

$$
\lambda_{0}<\frac{\epsilon}{F\left(r\left(x_{\epsilon}\right)\right)} \leq \epsilon
$$

Recall notations (6) and (7). Since

$$
\begin{aligned}
h_{\lambda_{0}}(x) & \geq h(x), \quad \forall x \in M, \\
h_{\lambda_{0}}\left(x_{\epsilon}\right) & =h\left(x_{\epsilon}\right),
\end{aligned}
$$

we have

$$
\begin{aligned}
& \nabla h_{\lambda_{0}}\left(x_{\epsilon}\right)=\nabla h\left(x_{\epsilon}\right), \\
& L h_{\lambda_{0}}\left(x_{\epsilon}\right) \geq \operatorname{Lh}\left(x_{\epsilon}\right) .
\end{aligned}
$$

Note that $\|\nabla r\|=1$. By (31), (33), and $G \geq 1$, the first equality of (35) yields

$$
\begin{aligned}
\left\|\nabla h\left(x_{\epsilon}\right)\right\| & =\left\|\lambda_{0} F^{\prime}\left(r\left(x_{\epsilon}\right)\right) \nabla r\left(x_{\epsilon}\right)\right\| \\
& <\frac{\epsilon}{F\left(r\left(x_{\epsilon}\right)\right)} \frac{F\left(r\left(x_{\epsilon}\right)\right)}{G\left(r\left(x_{\epsilon}\right)\right)} \leq \epsilon .
\end{aligned}
$$

Also, by (2), (31), (33), (36), $G \geq 1$, and $\widetilde{\Delta}_{x_{\varepsilon}} r \leq G$, the second inequality of (35) yields

$$
\begin{aligned}
& L h\left(x_{\epsilon}\right) \leq L h_{\lambda_{0}}\left(x_{\epsilon}\right)=\left.\sum_{l} a_{l l}\left(x_{\epsilon}\right) \frac{\partial^{2}}{\partial x_{l}^{2}} h_{\lambda_{0}}\right|_{x_{\epsilon}} \\
& +\left.\sum_{l} a_{l}\left(x_{\epsilon}\right) \frac{\partial}{\partial x_{l}} h_{\lambda_{0}}\right|_{x_{\epsilon}} \leq \lambda_{0}\left(F^{\prime}\left(r\left(x_{\epsilon}\right)\right) \tilde{\Delta}_{x_{\epsilon}} r\left(x_{\epsilon}\right)\right. \\
& \left.+F^{\prime \prime}\left(r\left(x_{\epsilon}\right)\right) \widetilde{\nabla}_{x_{\epsilon}} r\left(x_{\epsilon}\right) \cdot \nabla r\left(x_{\epsilon}\right)\right)+e_{1} \epsilon \\
& <\frac{\epsilon}{F\left(r\left(x_{\epsilon}\right)\right)}\left(\frac{F\left(r\left(x_{\epsilon}\right)\right)}{G\left(r\left(x_{\epsilon}\right)\right)} G\left(r\left(x_{\epsilon}\right)\right)\right. \\
& \left.+d_{1} \frac{F\left(r\left(x_{\epsilon}\right)\right)}{G\left(r\left(x_{\epsilon}\right)\right)^{2}}\right)+e_{1} \epsilon \leq \epsilon\left(1+d_{1}+e_{1}\right) .
\end{aligned}
$$

If we replace $\epsilon$ with $\epsilon\left(1+d_{1}+e_{1}\right)$, then the above inequality, (32), and (36) show that the point $x_{\epsilon}$ satisfies the conditions in Definition 1.

\section{Proof of Theorem 6}

The proof is similar to the method in [8]. Let $o \in M$ be a fixed point and $r(x)$ be the distance function from $o$. Define a function $u: M \rightarrow \mathbb{R}$ by

$$
u(x)=\int_{0}^{r(x)^{2}} G(s)^{-1} d s .
$$

Assume that a smooth complete Riemannian manifold satisfies assumption (10). Then we will prove that $u$ is an $L$-tamed exhaustion function. We consider two cases.

First Case. Assume that $o$ has no cut points in $M$.

By the definition, the function $u$ is an exhaustion function for $M$. We have to show that, for certain positive constants $C$ and $C_{1},\|\nabla u\|<C$ and $L u<C_{1}$ outside a ball of a certain radius with center $x_{\epsilon}$. Let $\phi(t)=\exp \left\{\int_{0}^{t} G(s)^{-1} d s\right\}$ and $B\left(x_{\epsilon}, r\right)=\left\{x \in M \mid \operatorname{dist}\left(x, x_{\epsilon}\right)<r\right\}$. Then $u(x)=$ $\log \phi\left(r(x)^{2}\right)$. By a direct calculation, one gets

$$
\nabla u=\nabla \log \phi\left(r^{2}\right)=2 r \nabla r \frac{\phi^{\prime}\left(r^{2}\right)}{\phi\left(r^{2}\right)}=2 r \nabla r G\left(r^{2}\right)^{-1} .
$$


By (12), there is a positive constant $C$ such that

$$
r^{2} \frac{G(r)}{G\left(r^{2}\right)}=r^{2} G(r) G\left(r^{2}\right)^{-1}<\frac{C}{4} .
$$

Then, for $r>1$, we obtain

$$
r G(r) G\left(r^{2}\right)^{-1}<r^{2} G(r) G\left(r^{2}\right)^{-1}<\frac{C}{4} .
$$

Moreover, by (11), we have

$$
\sup _{[0, \infty)} G(r)^{-1}=\left(\inf _{[0, \infty)} G(r)\right)^{-1} \leq 1 .
$$

By plugging (41) to (39), we have

$$
\|\nabla u\|<\frac{1}{2}\|\nabla r\| C G(r)^{-1} .
$$

Note that $\|\nabla r\|=1$. Applying (42) gives

$$
\|\nabla u\|<\frac{C}{2} .
$$

By (2) and (44), one gets

$$
\left\|\widetilde{\nabla}_{x_{\varepsilon}}^{1} u\right\|<e_{1} \frac{C}{2} .
$$

By assumption (11), we have

$$
\left(\frac{\phi^{\prime}\left(r^{2}\right)}{\phi\left(r^{2}\right)}\right)^{\prime}=\left(G\left(r^{2}\right)^{-1}\right)^{\prime}=-G\left(r^{2}\right)^{-2} G^{\prime}\left(r^{2}\right) \leq 0 .
$$

Because of the above inequality, $\left\|\widetilde{\nabla}_{x_{\varepsilon}} r\right\| \leq d_{1}$, (41), and (42), we have for $r>1$

$$
\begin{aligned}
\widetilde{\Delta}_{x_{\epsilon}} u= & \widetilde{\Delta}_{x_{\epsilon}} \log \phi\left(r^{2}\right) \\
= & 4 r^{2}\left(\frac{\phi^{\prime}\left(r^{2}\right)}{\phi\left(r^{2}\right)}\right)^{\prime}\left\|\widetilde{\nabla}_{x_{\epsilon}} r\right\|^{2} \\
& +2 G\left(r^{2}\right)^{-1}\left(\left\|\widetilde{\nabla}_{x_{\epsilon}} r\right\|^{2}+r \widetilde{\Delta}_{x_{\epsilon}} r\right) \\
\leq & 2 G\left(r^{2}\right)^{-1}\left(\left\|\widetilde{\nabla}_{x_{\epsilon}} r\right\|^{2}+r \widetilde{\Delta}_{x_{\epsilon}} r\right) \\
\leq & 2 r G\left(r^{2}\right)^{-1}\left(d_{1}^{2} r^{-1}+\widetilde{\Delta}_{x_{\epsilon}} r\right)
\end{aligned}
$$

$$
\begin{aligned}
& <\frac{C}{2} G(r)^{-1}\left(d_{1}^{2} r^{-1}+\widetilde{\Delta}_{x_{\epsilon}} r\right) \\
& <\frac{C}{2} d_{1}^{2}+\frac{C}{2} G(r)^{-1} \widetilde{\Delta}_{x_{\epsilon}} r .
\end{aligned}
$$

By our assumption (10), there exits $r_{0}>1$ such that

$$
\widetilde{\Delta}_{x_{\epsilon}} u<\frac{C}{2} d_{1}^{2}+\frac{C}{2} \quad \text { on } M \backslash B\left(x_{\epsilon}, r_{0}\right)
$$

Thus, by (45) and (48), we have

$$
\begin{array}{r}
L u=\widetilde{\Delta}_{x_{\varepsilon}} u+\widetilde{\nabla}_{x_{\epsilon}}^{1} u<\frac{C}{2}\left(d_{1}^{2}+1+e_{1}\right) \\
\quad \text { on } M \backslash B\left(x_{\epsilon}, r_{0}\right) .
\end{array}
$$

If we replace $(C / 2)\left(d_{1}^{2}+1+e_{1}\right)$ with $C_{1}$, then $u$ satisfies the additional conditions for an $L$-tamed exhaustion function.

Second Case. Assume that the cut locus of $o$ is nonempty.

Let $x_{\epsilon}$ be a cut point of $o$ and let $F(t)=\log \phi\left(t^{2}\right)$ for $t>0$. We choose a point $\widehat{x_{\epsilon}}$ outside of cut locus of $o$ such that $\operatorname{dist}\left(x_{\epsilon}, \widehat{x_{\epsilon}}\right)<1$ and $r\left(\widehat{x_{\epsilon}}\right)>r\left(x_{\epsilon}\right)$. Denote by $B(y, r)=\{x \in M \mid \operatorname{dist}(x, y)<r\}$. Take $\eta, \delta>0$ such that $B\left(x_{\epsilon}, \eta\right) \cap B\left(\widehat{x_{\epsilon}}, \delta\right)=\emptyset$ and $B\left(\widehat{x_{\epsilon}}, \delta\right)$ does not have cut point of $o$.

Now, we present several functions to find an uppersupporting function for $u$.

For a neighborhood $\mathcal{U} \subset B\left(x_{\epsilon}, \eta\right)$, we define a smooth map $T: \mathcal{U} \rightarrow B\left(\widehat{x_{\epsilon}}, \delta\right)$ with $T_{x_{\epsilon}}\left(x_{\epsilon}\right)=\widehat{x_{\epsilon}}$, and it is translation sending $x_{\epsilon}$ to $\widehat{x_{\epsilon}}$ in a coordinate chart including both $B\left(x_{\epsilon}, \eta\right)$ and $B\left(\widehat{x}_{\epsilon}, \delta\right)$ and satisfying $r(T(x)) \geq r(x)$. Also, we define a $C^{2}$ function $\lambda$ such that $\lambda\left(x_{\epsilon}\right)=1, \nabla \lambda\left(x_{\epsilon}\right)=0, \Delta \lambda\left(x_{\epsilon}\right)=0$, and

$$
\lambda(x) r(T(x)) \geq r(x)+r\left(\widehat{x_{\epsilon}}\right)-r\left(x_{\epsilon}\right) \quad \text { on } \mathcal{U} \text {. }
$$

Since $r\left(\widehat{x_{\epsilon}}\right)>r\left(x_{\epsilon}\right)$ and $r \geq 0$, we get $\lambda(x)>0$. Finally, for $x \in \mathcal{U}$, we define a function

$$
H(x)= \begin{cases}N(x)+\left(\frac{1}{2}\right) F^{\prime \prime}\left(r\left(x_{\epsilon}\right)\right) \lambda(x)\left(r(T(x))-r\left(\widehat{x}_{\epsilon}\right)\right)^{2} & \text { when } F^{\prime \prime}\left(r\left(x_{\epsilon}\right)\right)>0, \\ N(x)-\left(\frac{1}{2}\right) F^{\prime \prime}\left(r\left(\widehat{x}_{\epsilon}\right)\right)\left(r(T(x))-r\left(\widehat{x}_{\epsilon}\right)\right)^{2} & \text { when } F^{\prime \prime}\left(r\left(x_{\epsilon}\right)\right)<0, \\ N(x)+\left(\frac{1}{2}\right) Q\left(r\left(x_{\epsilon}\right)\right)\left(r(T(x))-r\left(\widehat{x}_{\epsilon}\right)\right)^{2} & \text { when } F^{\prime \prime}\left(r\left(x_{\epsilon}\right)\right)=0,\end{cases}
$$


where $N(x)=-F^{\prime}\left(r\left(\widehat{x_{\epsilon}}\right)\right)\left(r(T(x))-r\left(\widehat{x_{\epsilon}}\right)\right)+$ $F^{\prime}\left(r\left(x_{\epsilon}\right)\right)\left(\lambda(x) r(T(x))-r\left(\widehat{x_{\epsilon}}\right)\right)$ and $Q\left(r\left(x_{\epsilon}\right)\right)=\sup \left|F^{\prime \prime}(t)\right|$ for $t \in\left(r\left(x_{\epsilon}\right)-1, r\left(x_{\epsilon}\right)+1\right)$. Note that we choose $\widehat{x_{\epsilon}}$ as close to $x_{\epsilon}$ such that $\operatorname{sign}\left[F^{\prime \prime}\left(r\left(\widehat{x_{\epsilon}}\right)\right)\right]=\operatorname{sign}\left[F^{\prime \prime}\left(r\left(x_{\epsilon}\right)\right)\right]$. Therefore, $H(x)-N(x) \geq 0$.

Let $v(x)=F(r \circ T(x))+F\left(r\left(x_{\epsilon}\right)\right)-F\left(r\left(\widehat{x_{\epsilon}}\right)\right)+H(x)$. Then one gets $v\left(x_{\epsilon}\right)=F\left(r\left(x_{\epsilon}\right)\right)=u\left(x_{\epsilon}\right)$. Because of the fact $F^{\prime}(r(x)) \nabla r(x)=\nabla u(x)=G\left(r(x)^{2}\right)^{-1} 2 r(x) \nabla r(x)$ and the inequality (41), we get

$$
0<F^{\prime}(r(x))=G\left(r(x)^{2}\right)^{-1} 2 r(x)<\frac{C}{2} G(r(x))^{-1} .
$$

Moreover, we have two inequalities; that is, for $x \in \mathcal{U}$,

first order term of $v(x)-u(x)=F^{\prime}\left(r\left(x_{\epsilon}\right)\right)$

$$
\cdot\left(\lambda(x) r(T(x))-r\left(\widehat{x_{\epsilon}}\right)-\left(r(x)-r\left(x_{\epsilon}\right)\right)\right) \geq 0,
$$

second order term of $v(x)-u(x)=H(x)-N(x)$

$\geq 0$.

Hence $v$ is an upper-supporting function for $u$ at the point $x_{\epsilon}$.

Since $\left.\nabla H\right|_{x_{\epsilon}}=\left.\nabla N\right|_{x_{\epsilon}},\left\|\left.\nabla \lambda\right|_{x_{\epsilon}}\right\|=0, \lambda\left(x_{\epsilon}\right)=1$, and $\| \nabla(r \circ$ $T) \|=1$, we have

$$
\begin{aligned}
& \left\|\left.\nabla v\right|_{x_{\epsilon}}\right\| \leq\left|F^{\prime}\left(r\left(x_{\epsilon}\right)\right)\right| \\
& \quad \cdot\left(\left\|\left.\nabla \lambda\right|_{x_{\epsilon}}\right\| r\left(\widehat{x_{\epsilon}}\right)+\left|\lambda\left(x_{\epsilon}\right)\right|\left\|\left.\nabla(r \circ T)\right|_{x_{\epsilon}}\right\|\right) \\
& \quad=\left|F^{\prime}\left(r\left(x_{\epsilon}\right)\right)\right|=\left\|\left.\nabla u\right|_{x_{\epsilon}}\right\|<\frac{C}{2} .
\end{aligned}
$$

By our assumption (2), the above inequality implies that

$$
\left\|\left.\widetilde{\nabla}_{x_{\epsilon}}^{1} v\right|_{x_{\epsilon}}\right\|<e_{1} \frac{C}{2}
$$

Notice that

$$
\left.\widetilde{\Delta}_{x_{e}}(r \circ T(x))\right|_{x_{\varepsilon}}=\left.\|D T\|^{2} \widetilde{\Delta}_{x_{e}} r\right|_{\widehat{x_{e}}}=\left.n \widetilde{\Delta}_{\widehat{x}_{\varepsilon}} r\right|_{\widehat{x}_{\varepsilon}},
$$

where $\operatorname{dim} M=n$. By a simple calculation, we have

$$
\begin{aligned}
& F^{\prime \prime}(r(x)) \nabla r(x) \\
& \quad=2 G\left(r(x)^{2}\right)^{-1}\left(-2 r(x)^{2} G\left(r(x)^{2}\right)^{-1}+1\right) \nabla r(x)
\end{aligned}
$$

and hence

$$
\begin{aligned}
F^{\prime \prime} & (r(x)) \\
& =2 G\left(r(x)^{2}\right)^{-1}\left(-2 r(x)^{2} G\left(r(x)^{2}\right)^{-1}+1\right) \\
& <2 G\left(r(x)^{2}\right)^{-1} .
\end{aligned}
$$

Using $\|\nabla(r \circ T)\|=1,\left\|\widetilde{\nabla}_{x_{\varepsilon}}(r \circ T)\right\| \leq d_{1},(52)$, (56), and (58), we have

$$
\begin{aligned}
\left.\widetilde{\Delta}_{x_{\epsilon}} v\right|_{x_{\epsilon}} \leq & d_{1}^{2} F^{\prime \prime}\left(r\left(\widehat{x_{\epsilon}}\right)\right)+\left.F^{\prime}\left(r\left(\widehat{x_{\epsilon}}\right)\right) \widetilde{\Delta}_{x_{\epsilon}}(r \circ T)\right|_{x_{\epsilon}}+\left.\widetilde{\Delta}_{x_{\epsilon}} H\right|_{x_{\epsilon}} \\
& \leq \begin{cases}\left.F^{\prime}\left(r\left(x_{\epsilon}\right)\right) \widetilde{\Delta}_{x_{\epsilon}}(r \circ T)\right|_{x_{\epsilon}}+d_{1}^{2}\left(F^{\prime \prime}\left(r\left(\widehat{x_{\epsilon}}\right)\right)+F^{\prime \prime}\left(r\left(x_{\epsilon}\right)\right)\right) & \text { if } F^{\prime \prime}\left(r\left(x_{\epsilon}\right)\right)>0, \\
\left.F^{\prime}\left(r\left(x_{\epsilon}\right)\right) \widetilde{\Delta}_{x_{\epsilon}}(r \circ T)\right|_{x_{\epsilon}} & \text { if } F^{\prime \prime}\left(r\left(x_{\epsilon}\right)\right)<0, \\
\left.F^{\prime}\left(r\left(x_{\epsilon}\right)\right) \widetilde{\Delta}_{x_{\epsilon}}(r \circ T)\right|_{x_{\epsilon}}+d_{1}^{2}\left(F^{\prime \prime}\left(r\left(\widehat{x_{\epsilon}}\right)\right)+Q\left(r\left(x_{\epsilon}\right)\right)\right) & \text { if } F^{\prime \prime}\left(r\left(x_{\epsilon}\right)\right)=0, \\
& <\left.\left(\frac{1}{2}\right) C G\left(r\left(x_{\epsilon}\right)\right)^{-1} n \widetilde{\Delta}_{\widehat{x_{\epsilon}}} r\right|_{\widehat{x_{\epsilon}}}+4 d_{1}^{2} G\left(r\left(x_{\epsilon}\right)^{2}\right)^{-1} .\end{cases}
\end{aligned}
$$

Let $2 a$ be the distance to a closest cut point of $o$. Because the point $x_{\epsilon}$ is a cut point of $o$, by (41) and (42), we get

$$
\begin{aligned}
2 a G\left(r\left(x_{\epsilon}\right)^{2}\right)^{-1} & \leq r\left(x_{\epsilon}\right) G\left(r\left(x_{\epsilon}\right)^{2}\right)^{-1} \\
& <\frac{C}{4} G\left(r\left(x_{\epsilon}\right)\right)^{-1} \leq \frac{C}{4}, \\
G\left(r\left(x_{\epsilon}\right)^{2}\right)^{-1} & <\frac{C}{8 a} .
\end{aligned}
$$

By plugging (62) to (60), our assumption (10) tells us that, for $r>1$,

$$
\left.\widetilde{\Delta}_{x_{\varepsilon}} v\right|_{x_{\varepsilon}}<\frac{C}{2} n+\frac{C}{2 a} d_{1}^{2}
$$

Therefore, by (55) and (63), we obtain, for $r>1$,

$$
\left.L v\right|_{x_{\varepsilon}}<\frac{C}{2}\left(n+\frac{d_{1}^{2}}{a}+e_{1}\right) .
$$

So $u$ satisfies the conditions for an $L$-tamed exhaustion function.

Altogether, we can conclude that $u$ must be an $L$-tamed exhaustion function for $M$. 


\section{Conflict of Interests}

The author declares that there is no conflict of interests regarding the publication of this paper.

\section{Acknowledgments}

The author would like to thank the referee for valuable comments and corrections. Also, the author thanks Professor G. P. Bessa for pointing out $[6,14]$.

\section{References}

[1] H. Omori, "Isometric immersions of Riemannian manifolds," Journal of the Mathematical Society of Japan, vol. 19, pp. 205214, 1967.

[2] S. T. Yau, "Harmonic functions on complete Riemannian manifolds," Communications on Pure and Applied Mathematics, vol. 28, pp. 201-228, 1975.

[3] Q. Chen and Y. L. Xin, "A generalized maximum principle and its applications in geometry," American Journal of Mathematics, vol. 114, no. 2, pp. 355-366, 1992.

[4] A. Ratto, M. Rigoli, and A. G. Setti, "On the Omori-Yau maximum principle and its applications to differential equations and geometry," Journal of Functional Analysis, vol. 134, no. 2, pp. 486-510, 1995.

[5] A. Borbély, "A remark on Omori-Yau maximum principle," Kuwait Journal of Science, vol. 39, no. 2, pp. 45-56, 2012.

[6] G. P. Bessa, S. Pigola, and A. G. Setti, "Spectral and stochastic properties of the $f$-laplacian, solutions of PDEs at infinity and geometric applications," Revista Matemática Iberoamericana, vol. 29, no. 2, pp. 579-610, 2013.

[7] H. L. Royden, "The Ahlfors-Schwarz lemma in several complex variables," Commentarii Mathematici Helvetici, vol. 55, no. 4, pp. 547-558, 1980.

[8] K.-T. Kim and H. Lee, "On the Omori-Yau almost maximum principle," Journal of Mathematical Analysis and Applications, vol. 335, no. 1, pp. 332-340, 2007.

[9] K. Hong and C. Sung, "An Omori-Yau maximum principle for semi-elliptic operators and Liouville-type theorems," Differential Geometry and its Applications, vol. 31, no. 4, pp. 533-539, 2013.

[10] S. Pigola, M. Rigoli, and A. G. Setti, "A remark on the maximum principle and stochastic completeness," Proceedings of the American Mathematical Society, vol. 131, no. 4, pp. 12831288, 2003.

[11] S. Pigola, M. Rigoli, and A. G. Setti, Maximum Principles on Riemannian Manifolds and Applications, vol. 882 of Memoirs of the American Mathematical Society, American Mathematical Society, 2005.

[12] K. Takegoshi, "A volume estimate for strong subharmonicity and maximum principle on complete Riemannian manifolds," Nagoya Mathematical Journal, vol. 151, pp. 25-36, 1998.

[13] G. Albanese, L. J. Alías, and M. Rigoli, "A general form of the weak maximum principle and some applications," Revista Matemática Iberoamericana, vol. 29, no. 4, pp. 1437-1476, 2013.

[14] G. P. Bessa and L. F. Pessoa, "Maximum principle for semielliptic trace operators and geometric applications," Bulletin of the Brazilian Mathematical Society, vol. 45, no. 2, pp. 243-265, 2014.

[15] P. Petersen, Riemannian Geometry, vol. 171 of Graduate Texts in Mathematics, Springer, New York, NY, USA, 1998. 


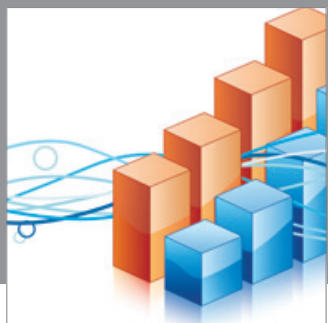

Advances in

Operations Research

mansans

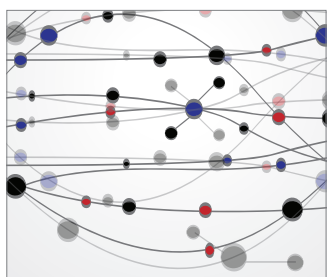

The Scientific World Journal
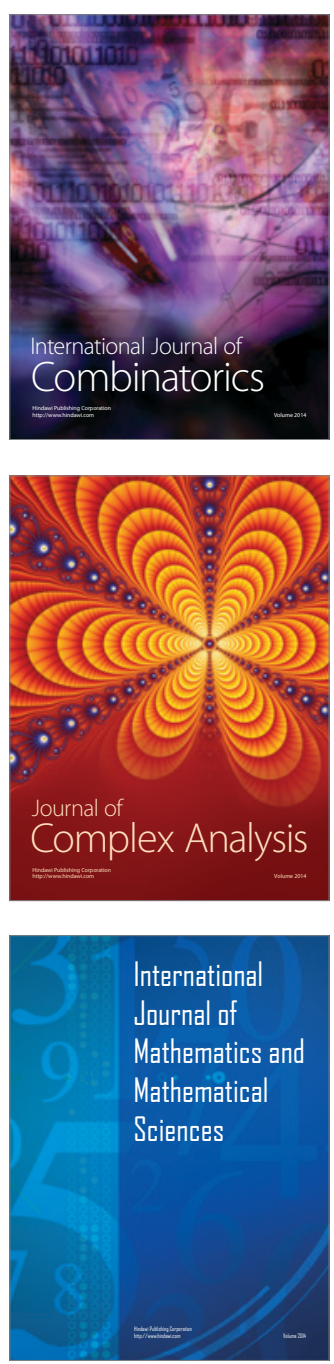
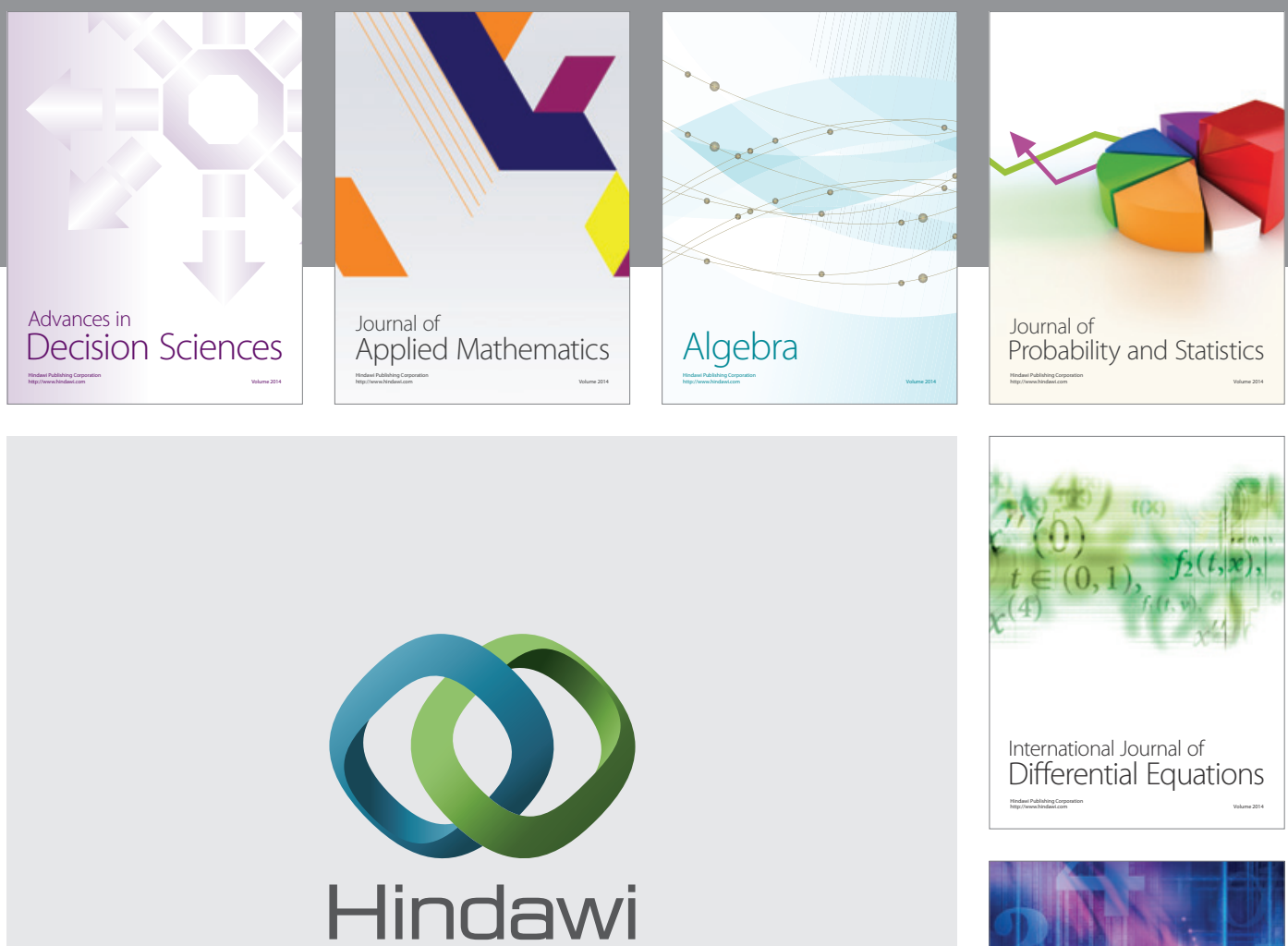

Submit your manuscripts at http://www.hindawi.com
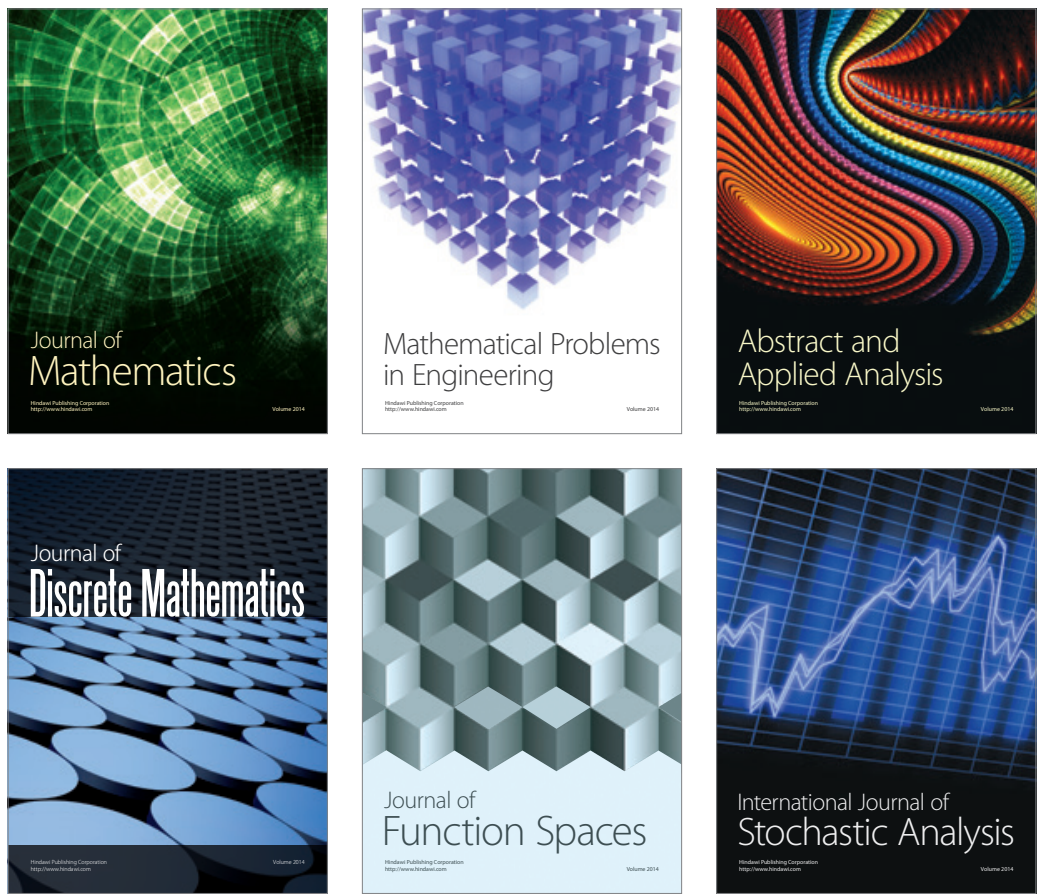

Journal of

Function Spaces

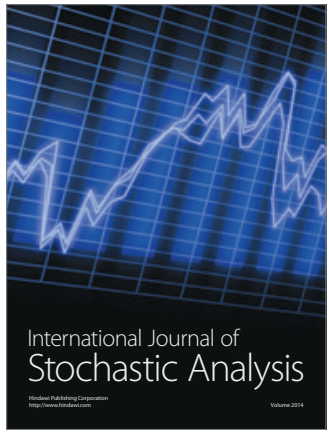

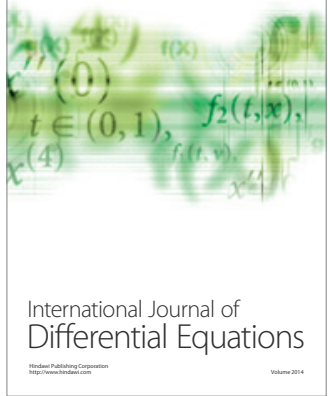
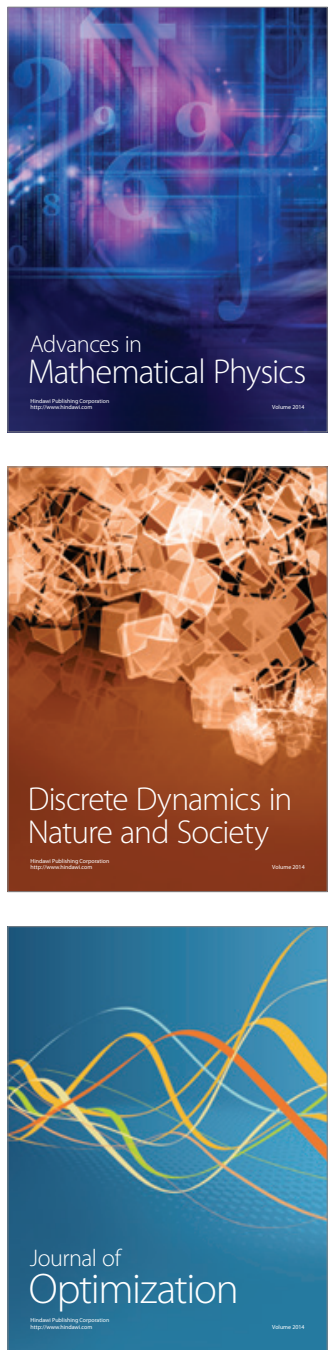